\title{
Innovative hybrid bonding method for strengthening reinforced concrete beam in flexure
}

\author{
Md. Moshiur Rahman, Mohd Zamin Jumaat*, Muhammad Ashiqur Rahman, Ismail M.I. Qeshta
}

Department of Civil Engineering, Faculty of Engineering, University of Malaya, 50603 Kuala Lumpur, Malaysia

H I G H L I G H T S

- The performance of hybrid bonding technique is better compared with the plate bonding method.

- With the increase of stiffness ductility was not affected in hybrid technique compared with plate bonding method.

- Bond performance between plate and concrete was increased by hybrid method.

\section{A R T I C L E I N F O}

Article history:

Received 27 December 2012

Received in revised form 8 November 2014

Accepted 27 December 2014

Available online 28 January 2015

Keywords:

Strengthening

Hybrid bond

Hybrid bond

Steel pla

Reinforced concrete

\begin{abstract}
A B S T R A C T
This paper presents the test results of the experimental behavior of reinforced concrete (RC) beams strengthened with the newly proposed hybrid bonding technique, which is the wise combination of plate bonding and near surface mounting (NSM) technique. Many studies have focused on the experimenta behavior of RC beams strengthened with externally bonded steel plates. However, the presence of high interfacial shear stresses at the end of the plate may reduce the resistance to failure of the strengthened structure. Recently, to a certain extent, the development of the near surface mounted strengthening technique can reduce the problem of this premature end debonding failure. However, the application of this method may be limited due to the absence of sufficient width and clear cover of the existing unstrengthened deficit beam. The hybridization bonding method could eliminate the limitations of these two methods to some extent. As data on the performance of structures strengthened with the hybrid bonding method are not available, the main purpose of this paper is to investigate the experimental behavior of RC beams strengthened with the hybrid bonding method. Seven medium-sized beams (one control and six strengthened) were tested. Steel plates and bars were used for strengthening. The failure load, failure mode, deflection, strain and cracking behavior are discussed. The test results confirm that the proposed hybrid bonding is the most effective strengthening technique without significantly hampering the ductility and its performance is $32 \%$ higher than that of corresponding plate bonding method through improvement of the bond performance.
\end{abstract}

(c) 2015 Elsevier Ltd. All rights reserved.

\section{Introduction}

Strengthening and upgrading of structures is required for several reasons including extension of design life, functional change mechanical damage and environmental effects, updated design requirements, and errors due to design and construction [1]. It is both environmentally and economically desirable to upgrade structures rather than rebuild them, particularly if rapid, effective and simple strengthening methods are available [2]. There are many methods available for strengthening existing deficient

\footnotetext{
* Corresponding author. Tel.: +60 37967 5203; fax: +60 379675318 E-mail address: zamin@um.edu.my (M.Z. Jumaat).
}

structures, among which, external plate bonding method and NSM techniques are the most popular.

Bonding steel plates or Carbon fiber reinforced plastic (CFRP) plates to the soffit of the RC structures to increase their strength or serviceability has been utilized worldwide since the late 1960 s. However, the development of high interfacial shear stresses at the plate ends could cause the premature debonding failure without utilizing the structure's full capacity. Many studies with the aim of finding solutions to reduce these stresses have been conducted [3]. One of the proposed solutions was to vary the thickness of the adhesive layer or the steel or FRP plate or the join geometry by tapering the plate [4]. However, although the use of geometrical changes in plate ends with taper form is an effective method to reduce the stresses in adhesive joints, it is quite

http://dx.doi.org/10.1016/j.conbuildmat.2014.12.081 


\begin{tabular}{|llll|}
\hline Symbols & & HS & hybrid strengthening \\
$a_{\mathrm{g}}$ & groove spacing & PS & plate bonding \\
$a_{\mathrm{e}}$ & edge clearance & LVDT & linear variable differential transducer \\
$\mathrm{CB}$ & control beam & & \\
\hline
\end{tabular}

complex, time consuming and requires higher cost. Another way to prevent debonding is to limit the design strain in FRP to a certain value, however, the results of this approach are highly conservative [5].

More recently, near-surface mounted (NSM) reinforcement has attracted an increasing amount of research as well as practical application because it is less prone to premature debonding [6]. However, it has some limitations in application. Sometimes, the width of the beam may not be wide enough to provide necessary edge clearance and clear spacing between two adjacent NSM grooves. ACI 440 recommends that the minimum edge clearance $\left(a_{\mathrm{e}}\right)$ and the clear spacing of the NSM groove $\left(a_{\mathrm{g}}\right)$ should be four and two times the groove depth. However, this recommendation has also been proven to be inadequate by De Lorenz [7] where one of the beams was strengthened with NSM spirally wound round bars with $a_{g}=30 \mathrm{~mm}$ (i.e. about 1.8 times the groove size and 3.6 times the bar diameter) and $a_{\mathrm{e}}=69 \mathrm{~mm}$ (i.e. about 4.3 times the groove size and 8.6 times the bar diameter), failed by debonding of the NSM bars involving the spalling of the concrete cover of the longitudinal steel reinforcement along the edges. In addition, the concrete cover should be higher to provide sufficient groove depth [8].

With respect to a number of drawbacks in the above two methods, this paper presents an idea to strengthen RC beams by combining the plate bonding method with the NSM strengthening technique and defines it as the hybrid bonding method. Through hybridization, both methods will complement each other and thereby possibly mutually overcome their limitations. It is known that the reduction in plate thickness decreases the magnitude of stress concentration at the plate extremities. Instead of tapering the plate, hybridization could make it possible to reduce the plate thickness by transferring a portion of the plate material from plate bonding to the NSM technique. Consequently, the size or number of NSM bars can also be reduced through shearing with plate bonding method, and, thus provide sufficient space for edge clearance and clear spacing of the groove.

In order to realize the above advantages of the hybrid bonding technique, the structural behavior of RC elements strengthened with the hybrid bonding method need to be fully characterized. Seven RC beams (one control six strengthened) were tested in this study. The examined variables were bonding technique, geometrical dimensions of the plate and NSM bar, number of grooves or NSM bars, performance of the tested beams and modes of failure are presented and discussed in this paper. Subsequently, the results of the experimental tests of the hybrid bonding method are compared with the corresponding plate bonding method. The test results show that the hybrid bonding method is better than the plate bonding method with respect to both strength and ductility performance, and, therefore it is a more effective and efficient strengthening technique.

\section{Theoretical advantages of the hybrid strengthening technique}

The main purpose of the hybrid strengthening technique is to improve the bond performance against premature failure between the existing concrete substrate and the applied strengthening plate and bar. Plate end debonding can probably be prevented through reduction of the interfacial stress. The reduction of interfacial shear and normal stress can be achieved in two ways - through hybridization of the plate bonding and NSM technique. One way is to reduce plate thickness by transferring some of the strengthening material from the plate to the NSM system. After transferring, the amount of strengthening material would be the same and produce similar flexure capacity of the beam. However, the magnitude of interfacial stress will be reduced due to the reduced plate thickness, because, the plate thickness is one of the most important parameters in reducing the interfacial stress. Fig. 1 shows the effect of the plate end thickness on the interfacial shear stresses in the CRFP strengthened beam [9].

Besides the experimental investigation, most of the codes of practice $[10,11]$ also recommended limiting the design strain on the plate to eliminate debonding. Other studies [12-15] have confirmed similar limits. However, in most cases, the design debonding strains are inversely proportional to plate thickness. For a fixed FRP ratio, debonding potential was shown to increase significantly with increasing FRP thickness [16]. Although the above study was based on the FRP strengthening system, it will be similarly applicable to the steel plate strengthening system.

A number of studies have focused on steel plate end debonding. Swamy et al. [17] showed that premature debonding of steel plates can effectively be avoided by ensuring that the width-thickness ratio of the plate is not less than 50. Swamy and Mukopadhyaya [18] have shown that this recommendation holds true for FRP plates when glass, glass-carbon, and aramid fibers are used. Oehlers [19] proposed a model based on interaction between the flexural and shear capacity of the beam where the ultimate debonding moment is also inversely proportional to plate thickness. Zibra's [20] model is a shear capacity based model where the debonding shear force decreases with steel plate thickness. To avoid debonding, Hassanen and Raoof [21] proposed design plate strain which is inversely proportional to the plate thickness. Therefore, reduction of the plate thickness is an effective way of preventing plate debonding.

Another way to reduce the interfacial stress is to increase the surface area. Since the hybrid strengthening technique is a combination of external plate bonding and the NSM technique, cutting a

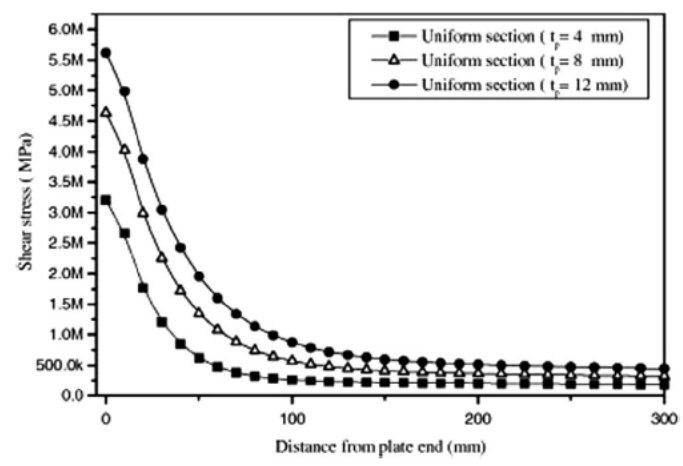

Fig. 1. The effect of the plate end thickness on the interfacial shear stresses [9]. 
groove for NSM strengthening obviously increase the surface area of the bonding face between the steel plate and the concrete substrate. Therefore, the interfacial stress, which is equal to the shear force divided by the corresponding surface area, will be reduced due to the increased surface area. Moreover, the addition of adhesive in the NSM groove in the hybrid strengthening system will further improve the bond performance between the strengthening steel plate and the concrete substrate. The mechanism for hybrid strengthening system is shown in Fig. 2.

\section{Experimental investigation}

An experimental program has been arranged to verify the effectiveness of the proposed hybrid bonding technique. Experimental data on load, deflection, strain and failure mode were obtained. The experimental program consisted of ten RC beams. In this section, a description of the material used, the test specimen and its different fabrication stages, the procedure used to strengthen the RC beams, the instrumentations and the test-setup are provided.

\subsection{Material used and its properties}

The materials used in this study and their properties were similar to the materials used by Alam [22] for the plate bonding method with only steel plate. This wa done to enable an effective comparison between the proposed hybrid strengthenin technique and the plate bonding method by maintaining the same the cross-sectional area thickness of the strengthening material.

\subsubsection{Concrete}

Ordinary Portland Cement (OPC) was used in casting the beams. The crushed granite (stone) was used as coarse aggregate, the maximum size of which was $20 \mathrm{~mm}$. Natural river sand was used as fine aggregate. Before casting, coarse an fine aggregates were washed with water and air dried in the concrete laboratory to get the saturated surface dry (SSD) condition. The concrete mix was designed for $30 \mathrm{MPa}$ concrete strength using the DOE method. The mix proportion adopted is as shown in Table 1. Material properties have effect on the compressive stret of concrete which can affect the bond properties and cracking behaviors.

\subsubsection{Concrete}

Ordinary Portland Cement (OPC) was used in casting the beams. The crushed granite (stone) was used as coarse aggregate, the maximum size of which was $20 \mathrm{~mm}$. Natural river sand was used as fine aggregate. Before casting, coarse and fine aggregates were washed with water and air dried in the concrete laboratory to get the saturated surface dry (SSD) condition. The concrete mix was designed for $30 \mathrm{MPa}$ concrete strength using the DOE method. The mix proportion adopted is as shown in Table 1. Material properties have effect on the compressive strength of concrete which can affect the bond properties and cracking behaviors.

\subsubsection{Reinforcement}

Four different diameters of steel bars were used in this research for preparation of the beam specimen. The main flexure reinforcement was two $12 \mathrm{~mm}$ diameter steel bars in the tension zone. The flexure reinforcement was bent 90 (ninety) degree at both ends to fulfill the anchorage criteria according to BS8110 [23]. Ten millimeters diameter plain bars were used as hanger bars in the shear span zone. Six millimeters diameter bars were used for stirrups. Six millimeters bars were also used for strengthening purpose in the hybrid strengthening system. Besides $6 \mathrm{~mm}$ used for strengthening purpose in the hybrid strengthening system. Besides $6 \mathrm{~mm}$
bar, $8 \mathrm{~mm}$ deformed bars were also used to strengthen RC beam particularly in the bar, $8 \mathrm{~mm}$ deformed bars were also used to strengthen RC
NSM groove. Properties of steel bars are shown in Table 2 .

\subsubsection{Steel plate}

Mild steel plates were used for external structural strengthening. Two different thickness of the steel plates were selected to strengthen the RC beam. $2 \mathrm{~mm}$ and $2.76 \mathrm{~mm}$ thick steel plate was used to investigate the effect of thickness on performance of hybridization. The width of all steel plates in this study is $73 \mathrm{~mm}$. Two different lengths of steel plate $(1650 \mathrm{~mm}$ and $1900 \mathrm{~mm})$ were used to observe the effect of plate length on the debonding behavior of the strengthened beam. For the first two strengthened beam, $2 \mathrm{~mm}$ thick steel plate along with $8 \mathrm{~mm}$ NSM bar (total area $196 \mathrm{~mm}^{2}$ ) was used to maintain the approximately same cross-sectional area $\left(201 \mathrm{~mm}^{2}\right)$ with the external plate bonding system tested by Alam [22] where the plate thickness is $2.76 \mathrm{~mm}$ and the width of plate is $73 \mathrm{~mm}$ as shown in Table 3. The yield strength and modulus of elasticity of the steel plate were $320 \mathrm{MPa}$ and $200 \mathrm{GPa}$ respectively.

\subsubsection{Epoxy}

Sikadur ${ }^{\$ 8} 30$ was used as the adhesive to ensure proper bonding between the concrete substrate and strengthening materials. The bond strength of epoxy on steel is $21 \mathrm{~N} / \mathrm{mm}$ according to German standard for pull-out test of adhesion DIN EN 24624 [24] and on concrete it is $4 \mathrm{~N} / \mathrm{mm}$. The average compressive, tensile
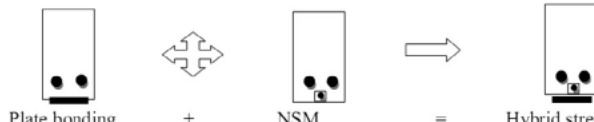

Plate bondin and shear strength of this adhesive are $80 \mathrm{MPa}, 15 \mathrm{MPa}$ and $25 \mathrm{MPa}$ respectively. It was also used to fill the NSM groove in the hybrid bonding technique. The bond strength of the epoxy plays an important role for evaluating the failure characteristics. The bond strength should be sufficient so that there is no failure between the surface of plate and epoxy.

\subsection{Beam design and preparation}

All beam specimens were $2300 \mathrm{~mm}$ long, $125 \mathrm{~mm}$ wide and $250 \mathrm{~mm}$ deep. Details of the reinforcement are shown in Fig. 3. The shear reinforcement was symmetrically placed in the shear span. A typical concrete cover of $30 \mathrm{~mm}$ was used. The concrete cover was measured from the center of flexural bar to bottom face of concrete. Sufficient shear reinforcement was provided to prevent sudden shear failure. The partial data collected from Alam [22] are presented in Table 3 for comparison and the control beams are the same for both study(Alam's and present).

The cement, sand, coarse aggregate and water with proper amounts were mixed using laboratory drum mixer. Steel moulds were used for casting. Before placing the concrete the steel moulds were cleaned and greased. After placing, the concrete was compacted using a poker vibrator. The beams were cast in three layers, where each layer was compacted using the poker vibrator to ensure adequate compaction. During the vibration process, each penetration was made at a reasonable distance from each other to avoid the bleeding and segregation of the concrete. Concrete was cast manually with different batch of mixing. These precaution measures were considmanually with different batch of mixing. These precaution measures were consid-
ered to get uniform strength according to mix design. If the beam strength varies much the analysis will be more difficult

\subsection{Strengthening of $R C$ beams}

\subsubsection{Surface preparation}

For the surface preparation, the concrete and steel plate requires special attention for proper bonding between the steel plate and concrete substrate. All oily, loose, dirty and any other foreign particles should be removed from the bonding surface of the concrete beams. In addition, the texture of the coarse aggregate of concrete beam should be exposed. Accordingly, the bonding faces of all the concrete beams were roughened using scabbler machine to expose the texture of the coars aggregate. The surface preparation of the concrete beam using scabbler machine is shown in Fig. 4.

\subsubsection{Groove cutting}

For the surface preparation, the concrete and steel plate requires special attention for proper bonding between the steel plate and concrete substrate. All oily, loose, dirty and any other foreign particles should be removed from the bonding surface of the concrete beams. In addition, the texture of the coarse aggregate concrete beam should be exposed. Accordingly, the bonding faces of all the concrete beams were roughened using scabbler machine to expose the texture of the coarse aggregate. The surface preparation of the concrete beam using scabbler machine is shown in Fig. 4.

\subsubsection{Groove cuttin}

Since the hybrid bonding method is a combination of external plate bondin and the NSM technique, grooves were first cut into the concrete cover of an RC element and the FRP reinforcement was bonded therein with appropriate groove filler. A special concrete saw was used to cut the grooves at the bottom surface of the beam. The groove dimensions were $10 \mathrm{~mm}$ wide by $15 \mathrm{~mm}$ deep. The specimen with one groove, the position of groove is on center of beam width at bottom. The specimen with two grooves, the groove spacing is $50 \mathrm{~mm}$. Therefore edge clearance will be $32.5 \mathrm{~mm}$. The length of groove and NSM steel bar is similar and equal plate length. The prepared specimens, after groove cutting and surface preparation for strengthening, are shown in Fig. 5.

\subsubsection{Placement of steel bar and plate}

The properly mixed Sikadur was then spread over the bonding area of the concrete surface. The groove was half filled with epoxy and the steel bars were placed and pressed into the centre of the groove. Then, the remaining space in the groove was completely filled with epoxy. The epoxy was levelled with a spatula and cured for at least 7 days before testing. The Sikadur was also applied with a special "dome" shaped spatula onto the bonding face of the strengthening plates. The plates were then placed on the prepared concrete beam surface. The properly placed plates were gently pressed using a rubber roller to remove the void in the interface area. NSM bars have the same length as the plate's length. After fixin the plates, the prepared beams were kept aside at least 7 days for proper curing.

\subsection{Instrumentation and test setup}

A Linear Variable Differential Transducer (LVDT) with working transverse rang of $50 \mathrm{~mm}$ was used to measure the deflection of the beam at mid span. The tran ducer was connected to a portable data logger to record the reading of the deflection of the beam during the test. In addition to the LVDT, the deflection at the mid span was also measured from the actuator vertical movement of the INSTRON (t) LVDT after initiating the failure of the beams. This information is useful to understand the deformability or ductility behavior of both the strengthened and un-strengthened beams.

Electrical resistance strain gauges measured the strains in the steel bar, steel plate or CFRP plate and the concrete. Two $5 \mathrm{~mm}$ strain gauges were attached to the middle of the internal main reinforcing bars to record the tension strain. Silicon was applied on the strain gauge as well as bare wire for proper water sealing. Two $30 \mathrm{~mm}$ strain gauges were placed at the middle of the top face of the concrete beam and bottom of the strengthening steel/FRP plate to measure the concrete compressive and plate tensile strain. The data logger was also connected with the digital

Fig. 2. Hybrid strengthening system. 
Table 1

Concrete mix design.

\begin{tabular}{|c|c|c|c|c|c|c|}
\hline \multirow{2}{*}{$\begin{array}{l}\text { Slump } \\
(\mathrm{mm})\end{array}$} & \multirow{2}{*}{$\begin{array}{l}\text { Water cement } \\
\text { ratio }\end{array}$} & \multicolumn{5}{|c|}{ Content $\left(\mathrm{kg} / \mathrm{m}^{3}\right)$} \\
\hline & & Water & er Cement & \multicolumn{2}{|c|}{$\begin{array}{l}\text { Coarse } \\
\text { aggregate }\end{array}$} & $\begin{array}{l}\begin{array}{l}\text { Fine } \\
\text { aggregate }\end{array} \\
\end{array}$ \\
\hline $30-60$ & 0.65 & 208 & 320 & 735 & & 1140 \\
\hline \multicolumn{7}{|c|}{$\begin{array}{l}\text { Table } 2 \\
\text { The properties of steel bar. }\end{array}$} \\
\hline $\begin{array}{l}\text { Bar diameter } \\
(\mathrm{mm})\end{array}$ & $\begin{array}{l}\text { Yield strength } \\
(\mathrm{MPa})\end{array}$ & \multicolumn{3}{|c|}{$\begin{array}{l}\text { Ultimate strength } \\
(\mathrm{MPa})\end{array}$} & \multicolumn{2}{|c|}{$\begin{array}{l}\text { Modulus of } \\
\text { elasticity (GPa) }\end{array}$} \\
\hline 6 & 580 & & 650 & & 200 & \\
\hline 8 & 551 & & 641 & & 200 & \\
\hline 10 & 520 & & 572 & & 200 & \\
\hline 12 & 551 & & 641 & & 200 & \\
\hline
\end{tabular}

Table 3

Load behavior of plate bonding system [22].

\begin{tabular}{llllll}
\hline $\begin{array}{l}\text { Beam } \\
\text { ID }\end{array}$ & $\begin{array}{l}\text { Plate dimension (each in } \\
\text { mm }) \\
(\text { length * width * thickness) }\end{array}$ & $\begin{array}{l}\text { First } \\
\text { crack } \\
\text { load } \\
(\mathrm{kN})\end{array}$ & $\begin{array}{l}\text { Bar } \\
\text { yield } \\
\text { load } \\
(\mathrm{kN})\end{array}$ & $\begin{array}{l}\text { Failure } \\
\text { load } \\
(\mathrm{kN})\end{array}$ & Mode of failure \\
\hline PS1 & $1900 * 73 * 2.76$ & 26 & 104 & 104 & $\begin{array}{l}\text { Cover separation } \\
\text { followed by } \\
\text { flexure-shear }\end{array}$ \\
PS2 $1600 * 73 * 2.76$ & 35 & 85 & 85 & $\begin{array}{l}\text { crack } \\
\text { Cover separation } \\
\text { followed by } \\
\text { flexure-shear } \\
\text { crack }\end{array}$ \\
\hline
\end{tabular}

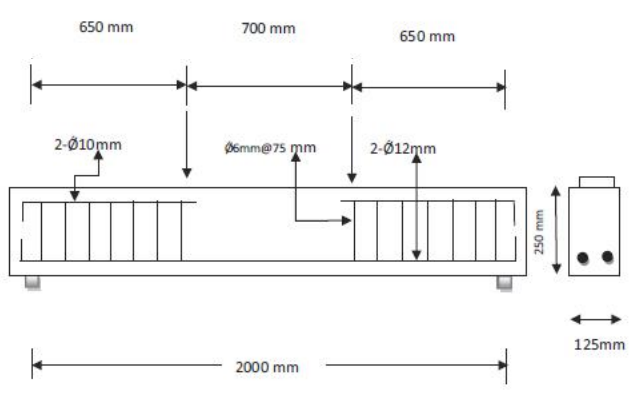

Fig. 3. Details of beam specimen.

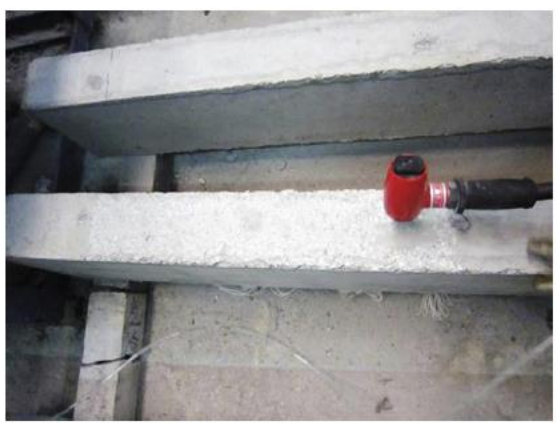

Fig. 4. Surface preparation of the concrete beam.

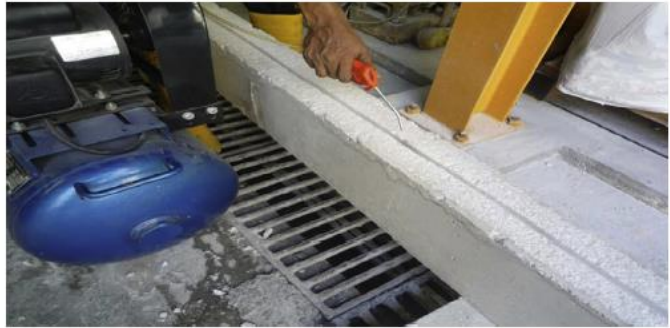

Fig. 5. The prepared specimen for strenthening.

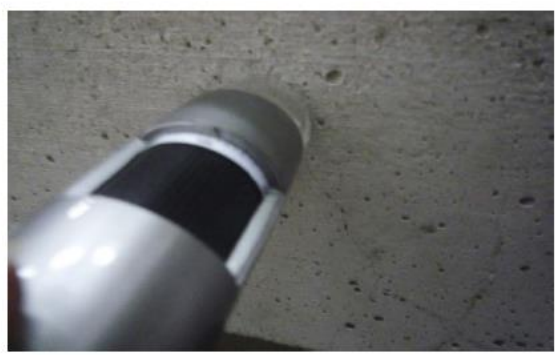

Fig. 6. Measuring crack width using Dino-lite digital microscope.

controller of the testing machine and strain gauges attached to the beams, for collecting the real-time loading and strains at the top concrete surface and bottom plate surface. The readings were scanned at a time interval of 1 (one) s.

A Dino-lite Digital Microscope was used to measure the cracks width of the concrete beams during the test which is shown in Fig. 6. By this device, the crack width can be measured up to $0.001 \mathrm{~mm}$. The lens was adjustable to obtain the sharpest picture of the cracks, and, consequently, the crack width can be estimated accurately. However, the cracks spacing along the beam length has to be measured manually.

All beams were tested under four-point bending. The tests were conducted with a closed-loop hydraulic Instron Universal testing machine. All specimens were simply supported and were subjected to two-point loading. The span was $2000 \mathrm{~mm}$ and the distance between the two loading points of the spreader beam was $700 \mathrm{~mm}$. The resulting shear span/depth ratio was 3.0. For the monotonic load test the actuator was loaded and moved down at a low rate so that reading from the data logger ator was loaded and moved down at a low rate so that reading from the data logger
and visibility of crack could be done easily. The test matrix is shown in Table 4.

\section{Results and discussion}

\subsection{Experimental behavior of hybrid strengthened beams}

\subsubsection{Load carrying capacity and failure mode}

Table 5 shows a summary of the flexural behavior of the all test beams in terms of load at first crack, yield load, flexural loading capacity and failure mode. As shown in Table 5, the addition of the steel bar and steel plate increases the ultimate moment capacity by $65 \%, 32 \%, 27 \%, 35 \%, 37 \%$ and $63 \%$ for HS1, HS2, HS3, HS 4 , HS5 and HS6, respectively, compared to the control beam. The yield load of the beam also increased after strengthening. Experimental result showed that the load at first crack of the strengthened beams increased most significantly compared to that of the control beam.

The failure modes of all beams are shown in Figs. 7-13. The failure modes of all the strengthened beams are found to be very close to each other. Concrete-cover separation is the most commonly reported mode of failure. This type of failure is generally demonstrated by a crack formed in the concrete at or near the plate end, propagating to the level of tension reinforcement and then

\section{Link to Full-Text Articles :}

